\title{
Summary Measures of Predictive Power Associated with Logistic Regression Models of Disease Risk
}

\author{
G. Hughes, ${ }^{1, \dagger}$ R. A. Choudhury, ${ }^{2}$ and N. McRoberts ${ }^{3}$ \\ ${ }^{1}$ Crop \& Soil Systems, SRUC, Edinburgh EH9 3JG, UK; ${ }^{2}$ Department of Plant Pathology, University of Florida, Gainesville 32611, U.S.A.; and \\ ${ }^{3}$ Department of Plant Pathology, University of California, Davis 95616, U.S.A. \\ Accepted for publication 9 December 2018.
}

\begin{abstract}
For an ordinary least-squares regression model, the coefficient of determination $\left(R^{2}\right)$ describes the proportion (or percentage) of variance of the response variable explained by the model, and is a widely accepted summary measure of predictive power. A number of $R^{2}$-analogues are available as summary measures of predictive power associated with logistic regression models, including models of disease risk. Tjur's $R^{2}$ and McFadden's $R^{2}$ are of particular interest in this context. Both of these metrics have transparent derivations, which reveal that they apply to different aspects of model evaluation. Tjur's $R^{2}$ is a measure of separation between (known) actual states (e.g., gold standard determinations of "healthy" or "diseased" status) whereas McFadden's $R^{2}$ is a measure of
\end{abstract}

ABSTRACT separation between predicted states (e.g., forecasts of disease status based on models of disease risk). This clarifies their interpretation in the context of evaluation of logistic regression models of disease risk. In addition, versions of both Tjur's $R^{2}$ and McFadden's $R^{2}$ may be obtained from analyses of disease risk that are not preceded by logistic regression analysis. Tjur's $R^{2}$ and McFadden's $R^{2}$ are shown to be useful, distinct summary measures of predictive power for epidemiological models of disease risk

Keywords: discrimination, logarithmic scoring rule, Nagelkerke's $R^{2}$, resolution.
At the core of this article is a discussion about ways to assess separation between levels of disease risk from epidemiological data. A common phytopathological context in which this problem arises is the application of logistic regression analysis to model disease risk from data. Here, we show how some summary statistics for logistic regression models can be interpreted in terms of separation between disease risk levels. However, the need to assess separation between levels of disease risk is wider in scope than the epidemiological application of logistic regression analysis. We also show how the summary statistics discussed in the context of logistic regression may be obtained more generally from analyses of disease risk that are not preceded by logistic regression analysis.

For an ordinary least-squares regression model, the coefficient of determination $\left(R^{2}\right)$ describes the proportion (or percentage) of variance of the response variable explained by the model, and is a widely accepted summary measure of predictive power. For logistic regression models, no single equivalent summary measure of predictive power seems to have achieved the same level of acceptance (Zheng and Agresti 2000). For example, the index of Logistic Regression from Introductory to Advanced Concepts and Applications (Menard 2010) mentions eight $R^{2}$-analogues, and even that list is not comprehensive. Here, our focus is on the kind of interpretation we can make of such $R^{2}$-analogues in the context of disease risk model evaluation. That is to say, the emphasis is on the requirements of model evaluation rather than on presentation of a list of $R^{2}$-analogues. We do not provide a tutorial on logistic regression (interested readers are referred to Yuen et al. [1996]), nor is it our intention to provide a review of the statistical literature on $R^{2}$-analogues.

Our initial objective is to present an overview of the role that $R^{2}$ analogues can play in the evaluation of epidemiological models of

${ }^{\dagger}$ Corresponding author: G. Hughes; E-mail: gareth.hughes@sruc.ac.uk

The author(s) declare no conflict of interest.

(c) 2019 The American Phytopathological Society disease risk. In pursuit of this objective, we discuss, in particular, $R^{2}$-analogues described by Tjur (2009) and by McFadden (1974). Tjur's $R^{2}$ is a relatively recent addition to the literature and, at the time of writing this article, does not seem to have had phytopathological application. McFadden's $R^{2}$ appears in the phytopathological literature referenced to its original source (Rimbaud et al. 2015) and also, on occasion, without such explicit acknowledgment. We also mention in passing what is probably the best known of the available $R^{2}$-analogues, described by Nagelkerke (1991) and applied by, for example, Pooler et al. (2002), Rossi et al. (2007, 2009), and Fourie et al. (2013), in addition to applications where the original source is not explicitly cited. There is no generally accepted terminology for what these $R^{2}$-analogues measure. Tjur (2009) refers to "explanatory power and ability to predict", McFadden (1974) to "variance explained", and Nagelkerke (1991) to the "proportion of explained variation". Menard (2010) refers to "explained variation", and usefully distinguishes between explained variation and goodness-of-fit (a distinction that we observe here, restricting our account to the former). We have adopted the term "predictive power" because it is the term used by Allison (2012), apparently the only mainstream text currently available that describes all three of the $R^{2}$-analogues discussed here in the context of model evaluation.

Our discussion is focused on the kind of probabilistic disease forecast often made in relation to a crop protection decision process. Tjur's (2009) analysis is restricted in application to decision processes with two actual states; thus, our discussion here is concerned with disease risk models based on binary logistic regression. In fact, this seems to cover the vast majority of published phytopathological applications to date.

We can write a generic logistic regression as

$$
\operatorname{logit}(p)\{=\ln [p /(1-p)]\}=f(\text { risk factors })
$$

in which the logit transformation of a probability $p$ (disease risk; which, depending on context, may refer, for example, to the probability of a disease outbreak or of the need for a control 
intervention) is modeled as a linear function of one or more explanatory variables, $f$ (risk factors). For analysis (Yuen et al. 1996), the response variable (left-hand side) is based on a retrospective gold-standard binary assessment of actual status of untreated crops (in the context of modeling a decision process). The corresponding data thus comprise two groups (denoted here as cases [c], numerically coded 1 and noncases [nc], coded 0 ). The explanatory variables (risk factors, right-hand side) may be quantitative (continuous or discrete) variables or qualitative (ordinal or nominal categorical) variables, or a combination of such variables, related to disease risk. More than one such combination may be put forward for evaluation, following routine statistical significance testing (further comment on this follows later).

The statistical evaluation of probabilistic forecasts may include the calculation of metrics defined conditionally on actual status, and also metrics defined conditionally on predicted status. In the meteorological literature (Joliffe and Stephenson 2012; Wilks 2011) these types of metric are referred to as measures of "discrimination" and of "resolution", respectively, and this terminology is adopted here. Discrimination and resolution are measures of separation. Tjur's $R^{2}$ is a measure of discrimination: separation between (known) actual states (e.g., gold standard determinations of "healthy" or "diseased" status). McFadden's $R^{2}$ is a measure of resolution: separation between predicted states (e.g., forecasts of disease status based on models of disease risk). We may now refine our objective as stated above: it is to present an overview of the role that $R^{2}$-analogues can play in the evaluation of epidemiological models of disease risk in terms of discrimination and resolution. Both discrimination and resolution are aspects of predictive power; each may be summarized by an appropriate $R^{2}$-analogue following logistic regression analysis. We also obtain versions of the $R^{2}$-analogues discussed here in terms of discrimination and resolution from analyses that do not require a preceding logistic regression, thus widening the scope of the summary statistics presented.

We illustrate our analysis with calculations based on an example dataset. The aim of our approach is to provide transparency-and therefore, hopefully, understanding-in relation to the way these metrics are used in summaries of the evaluation of epidemiological models of disease risk based on logistic regression analysis. The remainder of the article has the following format. An example dataset from the literature is briefly described and analyzed via binary logistic regression. The calculation of Tjur's $R^{2}$ as a measure of discrimination is described. The calculation of McFadden's $R^{2}$ as a measure of resolution is described. Nagelkerke's $R^{2}$ is discussed as a (rather inconvenient) measure of resolution. In the Discussion, we consider how Tjur's $R^{2}$ and McFadden's $R^{2}$ may be obtained from analyses of disease risk that are not preceded by logistic regression.

\section{ANALYSIS}

Data. For the purpose of illustration, we use an epidemiological dataset from a study of spinach downy mildew (SDM) caused by Peronospora effusa. The disease is a threat to sustainable production of organic spinach in California. The experimental program was described fully by Choudhury et al. (2016). Here, we consider a logistic regression analysis of SDM disease incidence on data derived from spore trapping for airborne inoculum. Spore trap data were recorded at successive lags before disease observations were made in nearby crops. The spore trap samples were used for extraction and assay of pathogen DNA, expressed as copy number of $P$. effusa ribosomal DNA. For analysis, these spore trap data were recorded on a natural logarithm (ln) scale. The present analysis uses $\ln$ (DNA copy number) at lag 4 as the single risk factor on the righthand side of equation 1. Disease incidence data were based on assessment of spinach crops for visual symptoms of SDM, as described in the account by Choudhury et al. (2016).
In total, $n=83$ data pairs were available for analysis. The modelfitting process yields a set of fitted probabilities $(\hat{p})$ which are plotted as a pair histograms, one for $\mathrm{c}$ and one for $\mathrm{nc}$, following Tjur (2009) (Fig. 1). On the horizontal axis, fitted probability represents an integrated risk score. Here, there is only a single risk factor in the regression model but, however complicated the $f$ (risk factors) is in terms of explanatory variables, we can always obtain and plot the set of fitted probabilities from the logistic regression analysis in this way (Tjur 2009).

Discrimination. The histograms shown in Figure 1 qualitatively illustrate separation between predicted outcomes for the two groups of subjects of known actual status (c and nc). Tjur (2009) quantifies this separation in terms of the coefficient of discrimination $(D)$, where

$$
D=\overline{\hat{p}}_{\mathrm{c}}-\hat{\hat{p}}_{\mathrm{nc}}
$$

in which $\overline{\hat{p}}_{\mathrm{c}}$ and $\overline{\hat{p}}_{\mathrm{nc}}$ denote the means of the histograms characterizing the frequency distributions of fitted probabilities for the c and nc groups, respectively, and $0<D<1$. Thus, $D$ characterizes the predictive power of a logistic regression model in terms of the separation between predictions for the $\mathrm{c}$ and nc groups, on a probability scale (i.e., discrimination). We calculate $D$ on the basis of the fitted probability values for individual subjects as calculated in the model output, not as binned for Figure 1. On this basis, $D=0.5646-0.4912=0.0734$ here (all numerical values are shown correct to four decimal places). Tjur (2009) goes on to show that $D$ is an analog of $R^{2}$ for use in logistic regression analysis.

The paired histograms shown in Figure 1 do not provide a basis for a diagnostic test until an appropriate threshold value of the variable on the horizontal axis has been defined. Thus, the next step toward a binary predictor of disease risk is to dichotomize the horizontal (fitted probability) axis of the paired histograms shown in Figure 1 with a single threshold (readers familiar with receiver operating characteristic curve analysis will likely recognize this procedure) (Twengström et al. 1998). Now, a prediction for an individual subject will be classified in one of two groups (denoted + for a predicted c and - for a predicted nc). The result is a 2-by-2 diagnostic table with individual subjects classified by actual status (c or nc) and by predicted status (+ or - ) (Table 1). In the table, A and $\mathrm{D}$ represent correct test outcomes for $\mathrm{c}$ (true positives) and nc (true negatives), respectively, while $\mathrm{B}$ and $\mathrm{C}$ represent incorrect test outcomes (false negatives and false positives, respectively). These quantities will vary depending on the placement of the threshold.

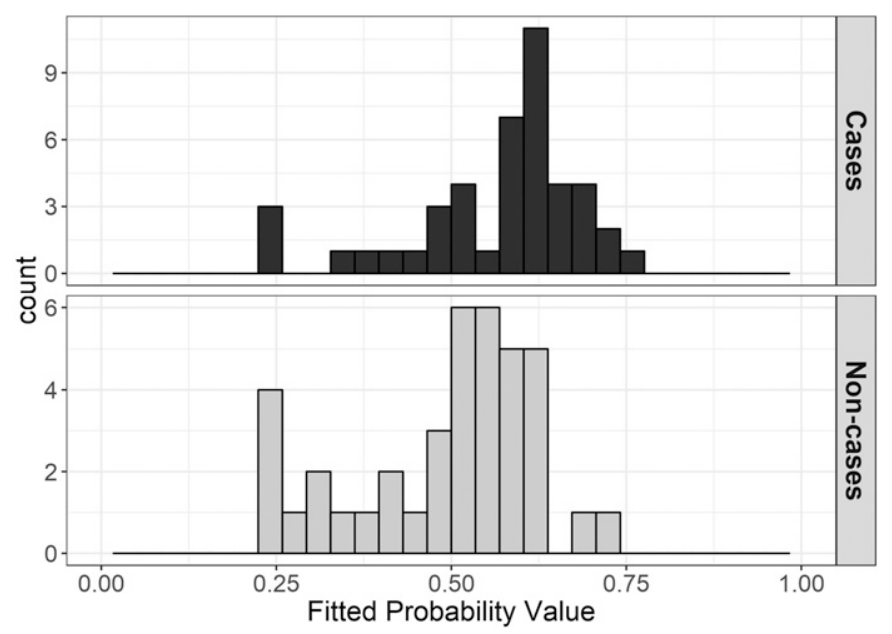

Fig. 1. Fitted probability values plotted separately for cases and noncases following binary logistic regression of spinach downy mildew disease incidence with $\ln$ (DNA copy number) at 4 lags as the single explanatory variable. 
The quantity $\mathrm{A} /(\mathrm{A}+\mathrm{B})$ is often referred to as "sensitivity" and the quantity $\mathrm{D} /(\mathrm{C}+\mathrm{D})$ as "specificity". Then, Youden's index for rating diagnostic tests $(J)$ (Youden 1950) is

$$
J=\text { sensitivity }+ \text { specificity }-1
$$

with $0<J<1$. In conditional probability notation, sensitivity is written as $\hat{p}_{+\mid \mathrm{c}}$ and specificity as $\hat{p}_{-\mid \mathrm{nc}}$; therefore, $J=\hat{p}_{+\mid \mathrm{c}}+\hat{p}_{-\mid \mathrm{nc}}-1=\hat{p}_{+\mid \mathrm{c}}-\hat{p}_{-\mid \mathrm{c}}$. While recognizing that there may be a range of determinants of the placement on the fitted probability axis of the threshold that defines the + and-groups, here, we take a simple approach and adopt the threshold that maximizes Youden's index (López-Ratón et al. 2014). There is a principled basis for this because Youden's index for a 2-by-2 diagnostic table is the same as discrimination measured by Tjur's $R^{2}$ for the logistic regression analysis of the table (Hughes 2017). Therefore, maximizing $J$ is the same as maximizing discrimination, which is reasonable at least as an initial approach to threshold specification. For the data in Table 1 , this approach leads to sensitivity $=0.6818$, specificity $=$ 0.6923 , and $J=0.3741$ (equation 2). This is a value of (Tjur's) $R^{2}$ that applies to discrimination of the diagnostic test characterized by Table 1.

Resolution. We now turn to separation as resolution. This analysis is based on a logistic regression of the SDM disease data in 2-by-2 table format (Table 1). As previously described, the response variable (actual disease status) is numerically coded 1 for $\mathrm{c}$ or 0 for nc. Now, however, the explanatory variable (predicted disease status) is a categorical variable, numerically coded 1 for a predicted c (+) or 0 for a predicted nc (-). As before, $n=83$ data pairs were available for analysis. From Table 1, we note that the Bayesian prior probability of c status is $\hat{p}_{\mathrm{c}}=44 / 83=0.5301$, and the corresponding Bayesian prior probability of nc status is $\hat{p}_{\mathrm{nc}}=39 / 83=0.4699$. These are the probabilistic predictions corresponding to the "null model", which does not include an analysis of risk factors. The model-fitting process then yields a set of four fitted probabilities: $\hat{p}_{\mathrm{c} \mid+}=0.7143$ (this is the Bayesian posterior probability of c status, given a modelbased prediction of c status), $\hat{p}_{\mathrm{nc} \mid+}=1-\hat{p}_{\mathrm{c} \mid+}=0.2857, \hat{p}_{\left.\mathrm{nc}\right|^{-}}=0.6585$ (this is the Bayesian posterior probability of nc status, given a modelbased prediction of nc status), and $\hat{p}_{\left.\mathrm{c}\right|^{-}}=1-\hat{p}_{\left.\mathrm{nc}\right|^{-}}=0.3415$. These are the probabilistic predictions corresponding to the "regression model", based on an analysis of risk factors.

Here, we illustrate model evaluation in terms of resolution via a logarithmic scoring rule (e.g., Dawid et al. 2016; see also Hughes and Burnett 2017 for a brief general introduction to scoring rules in a phytopathological context). The basic premise is that we give a score to each prediction, equal to the negative of the natural logarithm of the corresponding probability (the score is a penalty score; thus, better predictions get lower scores). For the null model, $\hat{p}_{\mathrm{c}}>0.5$; therefore, in the absence of any other evidence, we always predict "c". When a subject's actual status is c, the corresponding score is $-\ln \left(\hat{p}_{\mathrm{c}}\right)=0.6347$, and when the subject's actual status is nc, the corresponding score is $-\ln \left(\hat{p}_{\mathrm{nc}}\right)=0.7553$. Summing over subjects (44 c and $39 \mathrm{nc}$ ) to obtain the overall score for the null model, we calculate $S_{0}=57.3805$.

For the regression model, depending on the evidence provided by $f$ (risk factors), we may predict $\mathrm{c}$ or nc. Of the 42 predictions of $\mathrm{c}$

TABLE 1. A 2-by-2 diagnostic table for the spinach downy mildew (SDM) dataset $^{\mathrm{a}}$

\begin{tabular}{lcc}
\hline & \multicolumn{2}{c}{ Predicted disease status } \\
\cline { 2 - 3 } Actual disease status & - & + \\
\hline $\mathrm{c}$ & $\mathrm{B}=14$ & $\mathrm{~A}=30$ \\
$\mathrm{nc}$ & $\mathrm{D}=27$ & $\mathrm{C}=12$ \\
\hline
\end{tabular}

a Symbols: $\mathrm{c}=$ case, $\mathrm{nc}=$ noncase,$+=$ predicted case, and $-=$ predicted noncase. A, B, C, and D represent the observed frequencies in the two-way classification, as given for the SDM dataset. status, 30 were subjects with actual status c and 12 were subjects with actual status nc. The corresponding scores are $-\ln \left(\hat{p}_{\mathrm{c} \mid+}\right)=0.3365$ and $-\ln \left(\hat{p}_{\mathrm{nc} \mid+}\right)=1.2528$, respectively. Of the 41 predictions of nc status, 27 were subjects with actual status nc and 14 were subjects with actual status c. The corresponding scores are $-\ln \left(\hat{p}_{\left.\mathrm{nc}\right|^{-}}\right)=0.4177$ and $-\ln \left(\hat{p}_{\left.\mathrm{c}\right|^{-}}\right)=1.0745$, respectively. Summing over subjects to obtain the overall score for the regression model, we calculate $S=51.4494$. We note that the overall score for the regression model is lower than the overall score for the null model and, therefore, that the regression model represents an improved overall basis for predictions when compared with the null model (as it should). McFadden's (1974) $R^{2}$ is $1-\left(S / S_{0}\right)=0.1034$. For the logistic regression analysis, the scores $S_{0}$ and $S$ represent the negative log likelihoods for the null and regression models, respectively.

Steyerberg (2009) and Steyerberg et al. (2010) have pointed out that Nagelkerke's (1991) well-known $R^{2}$-analogue also arises via a logarithmic scoring rule. The corresponding analysis is less straightforward than that leading to McFadden's $R^{2}$. Here, after calculating the scores $S_{0}$ and $S$ as above, we may calculate an $R^{2}$ analogue from equation 2.44 of Maddala (1983) as

$$
1-\exp \left[-\left(\frac{2}{N}\right) \cdot\left(S_{0}-S\right)\right]=0.1332
$$

However, it is usually regarded as inconvenient that this quantity ranges from 0 to a maximum value of $<1$. Nagelkerke's (1991) response was to normalize Maddala's $R^{2}$ to obtain a 'maximum adjusted' $R^{2}$-analogue

$$
\frac{1-\exp \left[-\left(\frac{2}{N}\right) \cdot\left(S_{0}-S\right)\right]}{1-\exp \left[-\left(\frac{2}{N}\right) \cdot S_{0}\right]}=0.1332 / 0.7491=0.1778
$$

Nagelkerke's $R^{2}$ ranges from 0 to 1 , but as a result of the adjustment its value may be difficult to interpret in the comparative evaluation of models based on different explanatory variables (Menard 2010). Thus, application of Nagelkerke's $R^{2}$ is not necessarily straightforward when, as may often be the case in phytopathological applications, more than one combination of disease risk factors is put forward for evaluation following logistic regression analysis.

\section{DISCUSSION}

Discrimination and resolution represent two different perspectives on predictive power. We have seen that Tjur's $R^{2}$ is a discrimination metric, defined conditionally on actual disease status, whereas McFadden's $R^{2}$ (and Maddala's and Nagelkerke's) are resolution metrics, defined conditionally on the disease forecast. Readers should note that the terminology we have adopted here is not universal. As Yates (1990) pointed out, the descriptive terminology associated with the analysis of event judgments differs from field to field. For example, some care is required in relation to the clinical literature, where the main emphasis in model evaluation is often on assessment of the accuracy of predictions (including, but not restricted to, predictions based on logistic regression models) compared with known actual status; there, the term "discrimination" may be used to refer to both kinds of separation, and the term "resolution" may be used as a synonym for discrimination.

It is of interest to note that versions of both Tjur's $R^{2}$ and McFadden's $R^{2}$ may be obtained from analyses that are not preceded by a logistic regression analysis (it seems rather unlikely that anyone would obtain Nagelkerke's $R^{2}$ other than from the output of their logistic regression software). This provides us with a wider context for understanding the two metrics and their 
application. In a meteorological example where $\mathrm{c}$ and $\mathrm{nc}$ are "precipitation" and "no precipitation" events, respectively, section 8.4.5 in Wilks (2011) describes the "discrimination diagram" as an illustration of the separation between probabilistic predictions of these events for the two actual states. This is the same as Tjur's (2009) diagram of frequency distributions of fitted probabilities (Fig. 1, our phytopathological dataset), except that the horizontal axis is not based on the output of a logistic regression model but directly on the probabilistic predictions of the weather forecaster. The distance between the means of the two frequency distributions, referred to by Wilks as the "discrimination distance", is again used as a separation metric. Wilks' analysis provides a basis upon which we may assess the predictive power of probabilistic forecasts beyond the context of logistic regression while using the same performance metric as described by Tjur (2009) to describe discrimination.

For a 2-by-2 diagnostic table such as Table 1, Tjur's $R^{2}$ for logistic regression models is (as noted above) the same as Youden's index for rating diagnostic tests, calculation of which does not require the table to be based on the output of a logistic regression model. Thus, Youden's index provides a basis upon which we may assess the predictive power of probabilistic forecasts beyond the context of logistic regression while using the same performance metric to describe discrimination. Youden's index is already in wide application but not (until now) specifically with an interpretation as a summary measure of predictive power.

Turning now to resolution, Hauser (1978) provided an information theoretic interpretation of McFadden's $R^{2}$. Here, for brevity, we restrict discussion to the 2-by-2 diagnostic table and refer readers to Hughes and Burnett (2017) for full details of such analysis in a phytopathological context. The situation at the outset is that the actual status of a subject is either $\mathrm{c}$ or nc, with respective probabilities $\hat{p}_{\mathrm{c}}$ and $\hat{p}_{\mathrm{nc}}$. The uncertainty before we know the actual status is $-\sum_{j=\mathrm{nc}, \mathrm{c}} \hat{p}_{j} \cdot \ln \left(\hat{p}_{j}\right)$, referred to as the "entropy", here equal to 0.6913 . This is the amount of information that would be supplied by a notional perfect predictor. Even an imperfect predictor such as that characterized in Table 1 should supply some information and, thus, reduce this uncertainty. To quantify this, we calculate the "conditional entropy" (conditional on the predicted disease status), which is $-\sum_{i=-,+j=\mathrm{nc}, \mathrm{c}} \hat{p}_{j \mid i} \cdot \ln \left(\hat{p}_{j \mid i}\right)$, here equal to 0.6199 . Then, the difference $0.6913-0.6199=0.0715$ is the average reduction in uncertainty from use of the predictor in Table 1. This quantity, referred to as "expected mutual information", may also be interpreted as the average amount of information supplied by the predictor, its maximum value being the entropy. Then, expected mutual information is a proportion of the entropy, here $=0.0715$ / $0.6913=0.1034$. We can think of this as the proportion of uncertainty in disease status accounted for, on average, by application of the model. This quantity is identical to McFadden's $R^{2}$ as calculated via the logarithmic scoring rule above (in effect, we have carried out the same calculation but from a different perspective). Our information theoretic analysis of a 2-by-2 diagnostic table does not depend on a preceding logistic regression to provide the table. We note that Altman and Royston's (2000) PSEP is also a resolution metric for diagnostic tables that does not depend on a preceding logistic regression (for a 2-by-2 table, PSEP = $\hat{p}_{\mathrm{c} \mid+}-\hat{p}_{\mathrm{c} \mid-}$; here, PSEP $\left.=0.7143-0.3415=0.3728\right)$. The relationship between PSEP (measured directly on a probability scale) and expected mutual information (measured on an information scale) is illustrated by Hughes and Burnett (2017).

Typically, in descriptions of phytopathological models of disease risk based on logistic regression, little explicit interpretation is given to an $R^{2}$-analogue accompanying the analysis. An implicit interpretation in terms of some sort of equivalence with the $R^{2}$ for an ordinary least-squares regression model is perhaps intended in such cases. However, as we have shown here, Tjur's $R^{2}$ and McFadden's
$R^{2}$ are useful summary measures of predictive power that have clear interpretations relating to different aspects of probability separation, that have transparent derivations, and that are not necessarily restricted in application to the evaluation of models based on a preceding logistic regression analysis.

\section{LITERATURE CITED}

Allison, P. D. 2012. Logistic Regression Using SAS: Theory and Application, 2nd ed. SAS Institute Inc., Cary, NC, U.S.A.

Altman, D. G., and Royston, P. 2000. What do we mean by validating a prognostic model? Stat. Med. 19:453-473.

Choudhury, R. A., Koike, S. T., Fox, A. D., Anchieta, A., Subbarao, K. V., Klosterman, S. J., and McRoberts, N. 2016. Season-long dynamics of spinach downy mildew determined by spore trapping and disease incidence. Phytopathology 106:1311-1318.

Dawid, A. P., Musio, M., and Ventura, L. 2016. Minimum scoring rule inference. Scand. J. Stat. 43:123-138.

Fourie, P., Schutte, T., Serfontein, S., and Swart, F. 2013. Modeling the effect of temperature and wetness on Guignardia pseudothecium maturation and ascospore release in citrus orchards. Phytopathology 103:281-292.

Hauser, J. R. 1978. Testing the accuracy, usefulness, and significance of probabilistic choice models: An information-theoretic approach. Oper. Res. 26:406-421.

Hughes, G. 2017. Tjur's $R^{2}$ for logistic regression models is the same as Youden's index for a $2 \times 2$ diagnostic table. Ann. Epidemiol. 27:801-802.

Hughes, G., and Burnett, F. J. 2017. Evaluation of probabilistic disease forecasts. Phytopathology 107:1136-1143.

Joliffe, I. T., and Stephenson, D. B., eds. 2012. Forecast Verification: A Practitioner's Guide in Atmospheric Science, 2nd ed. John Wiley \& Sons, Ltd., Chichester, U.K.

López-Ratón, M., Rodríguez-Álvarez, M. X., Cadarso-Suárez, C., and Gude-Sampedro, F. 2014. OptimalCutpoints: An R package for selecting optimal cutpoints in diagnostic tests. J. Stat. Softw. 61:1-36.

Maddala, G. S. 1983. Limited-Dependent and Qualitative Variables in Econometrics. Cambridge University Press, Cambridge, U.K.

McFadden, D. 1974. Conditional logit analysis of qualitative choice behavior. Pages 105-142 in: Frontiers in Econometrics. P. Zarembka, ed. Academic Press, New York, NY, U.S.A.

Menard, S. 2010. Logistic Regression: From Introductory to Advanced Concepts and Applications. Sage, Thousand Oaks, CA, U.S.A.

Nagelkerke, N. J. 1991. A note on a general definition of the coefficient of determination. Biometrika 78:691-692.

Pooler, M. R., Jacobs, K. A., and Kramer, M. 2002. Differential resistance to Botryosphaeria ribis among Cercis taxa. Plant Dis. 86:880-882.

Rimbaud, L., Dallot, S., Delaunay, A., Borron, S., Soubeyrand, S., Thébaud, G., and Jacquot, E. 2015. Assessing the mismatch between incubation and latent periods for vector-borne diseases: The case of sharka. Phytopathology 105:1408-1416.

Rossi, V., Bolognesi, M., and Giosuè, S. 2007. Influence of weather conditions on infection of peach fruit by Taphrina deformans. Phytopathology 97: 1625-1633.

Rossi, V., Salinari, F., Pattori, E., Giosuè, S., and Bugiani, R. 2009. Predicting the dynamics of ascospore maturation of Venturia pirina based on environmental factors. Phytopathology 99:453-461.

Steyerberg, E. W. 2009. Clinical Prediction Models: A Practical Approach to Development, Validation, and Updating. Springer, New York, NY, U.S.A.

Steyerberg, E. W., Vickers, A. J., Cook, N. R., Gerds, T., Gonen, M., Obuchowski, N., Pencina, M. J., and Kattane, M. W. 2010. Assessing the performance of prediction models: A framework for traditional and novel measures. Epidemiology 21:128-138.

Tjur, T. 2009. Coefficients of determination in logistic regression models-A new proposal: The coefficient of discrimination. Am. Stat. 63:366-372.

Twengström, E., Sigvald, R., Svensson, C., and Yuen, J. 1998. Forecasting Sclerotinia stem rot in spring sown oilseed rape. Crop Prot. 17:405-411.

Wilks, D. S. 2011. Statistical Methods in the Atmospheric Sciences, 3rd ed. Academic Press, Oxford, U.K.

Yates, J. F. 1990. Judgment and Decision Making. Prentice Hall, Englewood Cliffs, NJ, U.S.A.

Youden, W. J. 1950. Index for rating diagnostic tests. Cancer 3:32-35.

Yuen, J., Twengström, E., and Sigvald, R. 1996. Calibration and verification of risk algorithms using logistic regression. Eur. J. Plant Pathol. 102:847-854.

Zheng, B., and Agresti, A. 2000. Summarizing the predictive power of a generalized linear model. Stat. Med. 19:1771-1781. 